\title{
Modelagem e Predição temporal de Parâmetros de Qualidade de Água usando Redes Neurais Profundas*
}

\author{
Anderson Almeida , Marcos Amaris , Bruno Merlin , Allan Veras ${ }^{1}$ \\ ${ }^{1}$ Universidade Federal do Pará \\ Núcleo de Desenvolvimento Amazônico de Engenharia, Tucuruí - Pará \\ \{andersonchico, bruno.merlin\}@gmail.com
\{amaris, allanveras
}

\begin{abstract}
The quality of the water is directly related to its level of pollution, and for that, monitoring is necessary to identify the physical, chemical, and biological characteristics, considering the current legislation. This article presents a comparison of the Long-Short Term Memory (LSTM) and Perceptron Multilayer (MLP) neural network models to predict the $\mathrm{pH}, \mathrm{OD}, \mathrm{BOD}$, Phosphorus, and Turbidity parameters of water quality. The error metrics RMSE and MSE were used, when the neural networks are configured with 10, 25, and 50 neurons. The LSTM network presented an average RMSE of 0.134, average MSE of 0.035 , and average MAPE of 13.49. The MLP network presented average RMSE 0.085, average MSE of 0.01, and average MAPE of 13.03. The results of the experiments aim to contribute to the process of monitoring water quality and to assist water management planning through the appropriate machine learning model for predicting parameters.
\end{abstract}

Resumo. A qualidade da água está diretamente relacionada com o seu nível de poluição, e para isso, é necessário o monitoramento para identificar as características físicas, químicas e biológicas, considerando a legislação vigente. Este artigo apresenta a comparação dos modelos de rede neural Long-Short Term Memory (LSTM) e Perceptron Multilayer (MLP) para predizer os parâmetros pH, OD, DBO, Fósforo e Turbidez da qualidade da água. Foram usadas as métricas de erro RMSE e MSE, quando as redes neurais são configuradas com 10, 25 e 50 neurônios. A rede LSTM apresentou um RMSE médio de 0.134, MSE médio de 0,035 e MAPE médio de 13,49. A rede MLP apresentou RMSE médio de 0,085, MSE médio de 0.01 e MAPE médio de 13.03. Os resultados do experimentos visam contribuir com o processo de monitoramento da qualidade da água e auxiliar o planejamento da gestão hídrica através do modelo de aprendizado de máquina adequado para predição dos parâmetros.

\section{Introdução}

A água é um recurso hídrico essencial para o consumo humano, para as atividades industriais e agrícolas, assim como para os ecossistemas vegetal e animal. A oferta de água é determinada pela dinâmica hídrica e socioeconômica das bacias dos rios, além das condições da qualidade da água.

*Esta pesquisa é parte do INCT da Internet do Futuro para Cidades Inteligentes, financiado por CNPq (proc. 465446/2014-0), Coordenação de Aperfeiçoamento de Pessoal de Nível Superior - Brasil (CAPES) - Código de Financiamento 001 e FAPESP (procs. 14/50937-1 e 15/24485-9). 
E o conhecimento dessa oferta é produzido pelo monitoramento hidrológico dos recursos hídricos superficiais sobre os parâmetros básicos da qualidade da água como $\mathrm{pH}$, temperatura, oxigênio dissolvido (OD), turbidez e condutividade elétrica [ANA 2018].

As análises preditivas sobre uma series temporais contribuem para a qualidade da tomada de decisão [Solanki et al. 2015]. A área da computação, que trata amplamente de análises preditivas é o aprendizado de máquina, que é uma sub-área da inteligência artificial aplicada, para adquirir conhecimento de forma automática [Monard and Baranauskas 2003]. Uma técnica de aprendizado de máquina baseada na simulação do cérebro humano são as Redes Neurais Artificiais (RNA). Diferentes e não poucas arquiteturas de RNA têm sido criadas nos últimos anos.

No presente trabalho será utilizada a arquitetura de redes neurais recorrentes LongShort Term Memory (LSTM) [Da Silva et al. 2010] e Multilayer Perceptron (MLP) para a modelagem e predição temporal de parâmetros de qualidade de água.

A análise preditiva realizada neste artigo é realizada utilizando dados de monitoramento dos parâmetros da qualidade da água em duas Unidades de Gerenciamento de Recursos Hídricos (UGRHI) no Estado de São Paulo. Essas UGRHI estão localizadas em áreas classificadas como industrializadas 1

Portanto, pretende-se comparar as predições dos modelos de rede neural LSTM e MLP, na predição dos parâmetros de qualidade de água pH (Potencial Hidrogeniônico), OD (Oxigênio Dissolvido), DBO (Demanda Bioquímica de Oxigênio), Fósforo e Turbidez. Essas predições foram comparadas temporalmente. Foram usadas três métricas de erro. Erro quadrático médio (MSE), raiz do erro quadrático médio (RMSE) e erro percentual absoluto médio (MAPE), quando as RNAs foram configuradas com diferentes números de neurônios.

Desta forma, este trabalho visa contribuir com o processo de monitoramento da qualidade das águas, avaliando as suas tendências e diagnósticos, tornando-se um subsídio para o planejamento da gestão hídrica em manter o equilíbrio entre o desenvolvimento econômico, demográfico e a disponibilidade hídrica para diversos tipos de usos.

Além da introdução, o artigo é estruturado da seguinte forma. Na Seção 2 são apresentados os conceitos dos parâmetros da qualidade da água e de redes neurais LSTM e MLP; Na Seção 3 trata-se dos trabalhos relacionados. A Seção 4 apresenta a metodologia usada no trabalho. Na Seção 5 são discutidos os resultados e finalmente na Seção 6 são apresentadas as conclusões e os trabalhos futuros.

\section{Conceitos}

Nesta seção serão apresentados os conceitos utilizados neste trabalho. Assim esclareceremos alguns fundamentos teóricos referentes aos parâmetros da qualidade da água e às redes neurais artificiais usadas nesse trabalho.

\subsection{Parâmetros da Qualidade da Água}

A qualidade da água é formada pelas características de natureza física, química e biológica, representadas por parâmetros que indicam a sua qualidade, de acordo com

\footnotetext{
${ }^{1}$ CETESB (2019). Infoáguas .https://sistemainfoaguas.cetesb.sp.gov.br/. [Online; De-zembro 7 de 2019
} 
o valor padrão estabelecido em lei para cada finalidade de uso [Derisio 2016]. Os valores dos parâmetros são conhecidos durante o monitoramento da qualidade da água, tornandose uma importante ação para a gestão dos recursos hídricos, pois possibilita a atualização das condições desses recursos, além de subsidiar a descoberta de conhecimento futuro dos valores dos parâmetros da qualidade das águas superficiais ou subterrâneas através da análise dos dados coletados durante uma regularidade temporal [Gastaldini et al. 2001]. A seguir serão descritos os parâmetros utilizados no presente estudo:

- Oxigênio Dissolvido (OD): O OD é um indicador primário da qualidade da água de um rio. O OD é importante para a avaliação da qualidade da água, pois é essencial para o metabolismo e a sobrevivência dos seres vivos de um corpo de água.

- Potencial Hidrogeniônico (pH): É um dos parâmetros mais utilizados na análise da qualidade da água, determinando se a água é ácida, cujos valores influenciam diretamente na fisiologia de várias espécies.

- Turbidez: É uma característica física que resulta na quantidade de luz refletida mediante a presença de material em suspensão nos corpos d'água [de Fravet and Cruz 2007].

- Fósforo : É uma parâmetro físico-químico que em grande concentração identifica a presença de poluição em um corpo d'água [Gastaldini and Teixeira 2001].

- Demanda Bioquímica de Oxigênio (DBO) É um parâmetro responsável pela identificação da quantidade de matéria orgânica biodegradável no corpo de água [Gastaldini and Teixeira 2001], portanto o aumento do nível de DBO indica a insuficiência de oxigênio, e consequentemente, extinção da vida aquática.

\subsection{Redes Neurais Artificiais}

As técnicas de regressão tentam relacionar um conjunto de observações com objetivo explicativo, que indica uma relação de causa/efeito, ou preditivo diante de novas observações. Há várias técnicas que implementam a regressão como as redes neurais, máquinas de vetores de suporte, árvores de decisão, entre outras [Goldschmidt and Passos 2005].

A rede neural artificial simula a atividade cerebral humana para resolver problemas particulares. Dois aspectos são semelhantes ao cérebro: conhecimento adquirido por processo de aprendizagem, que é o ajuste ordenado dos pesos sinápticos, e a conexão entre neurônios para armazenar o conhecimento [Haykin 2007]. É estruturada com sinais de entrada $(x)$ que são as variáveis da aplicação; os pesos sinápticos $(w)$ que ponderam cada sinal de entrada; o combinador linear soma os valores dos sinais de entrada com os pesos sinápticos; a função de ativação $(g)$ define o intervalo de valores na saída do neurônio; e a saída (y) apresenta o valor final da rede neural [Da Silva et al. 2010].

\subsubsection{Rede Neural MLP}

Segundo [Da Silva et al. 2010], as redes perceptron multicamadas pertencem a arquitetura feedforward de múltiplas camadas, são caracterizadas pela presença de no mínimo uma camada oculta entre as camadas de entrada e saída, com treinamento supervisionado. A configuração topológica quanto a quantidade de camadas ocultas e ao número de neurônios dependem de fatores como a classe do problema, das amostras disponibilizadas no treinamento, dos valores de entrada e dos pesos [Da Silva et al. 2010]. 
O processo de treinamento da rede perceptron multicamadas apresenta duas fases: a propagação adiante (forward) e a propagação reversa (backward), por isso, o referido processo de treinamento é chamado de Backpropagation. Na fase propagação adiante os sinais de entrada são propagados pelas camadas até a camada de saída, sem alteração dos pesos sinápticos gerando as respostas da rede, que serão comparadas com as respostas desejadas, calculando o erro. Na propagação reversa, o sentido é inverso da propagação adiante e o erro calculado na primeira fase é utilizado como ajuste dos pesos sinápticos e limiares durante cada época configurada [Da Silva et al. 2010].

\subsubsection{Rede Neural LSTM}

Entre as arquiteturas de redes neurais usadas no aprendizado profundo, podemos citar a LSTM que tem o objetivo de reparar a falta de memória de longo prazo da rede neural, retendo as principais informações dos sinais de entrada [Bandara et al. 2020], e proporciona a persistência dessas informações realizada com laços de repetição. A estrutura da rede neural LSTM apresenta um mecanismo chamado de comportas que regulam o fluxo da informação nas células de memória [Olah 2015]. Resumidamente, a LSTM é composta pela cadeia de células de memória, que indica o estado das células, e as comportas, que manipulam as informações.

\section{Trabalhos Relacionados}

Diversos trabalhos abordam o uso de redes neurais para predição de parâmetros da qualidade. No estudo de [Sarkar and Pandey 2015], são aplicadas redes neurais perceptron multicamadas com algoritmo de retropropagação de erro (Backpropagation) para simular os valores do OD no rio Yamuna na cidade de Mathura na Índia. No experimento desenvolveram 3 redes com 14, 10 e 5 variáveis de entrada. O conjunto de dados utilizados no experimento trata-se do período de 1990 a 1996, com informações mensais sobre a vazão de fluxo e de parâmetros como temperatura, pH, DBO e OD. Para validação do desempenho do modelo foram utilizados avaliação estatística como erro quadrático médio (RMSE), coeficiente de correlação (R) e coeficiente de determinação (DC). Os resultados foram que a rede neural é eficiente para simular valores de OD, principalmente, quando há 10 variáveis para a entrada na rede neural

No estudo de [Sahoo et al. 2015], foi utilizado o Adaptative Neural Fuzzy Inference System (ANFIS) e análise de componentes principais para prever o índice da qualidade da água (IQA), com 11 parâmetros (pH, DO, DBO, Condutividade, Nitrato-N, NH4-N, COD, TC, FC, TA de $\mathrm{CaCO} 3$ e TH de CaCO3) da qualidade da água como entrada de dados. Para validação do treinamento e testes foi utilizado o erro percentual médio absoluto e conclui que o modelo ANFIS quando utiliza 4 variáveis como entrada de dados torna-se eficiente na previsão.

No estudo de [Solanki et al. 2015], utilizou-se o modelo de aprendizagem profunda Deep Belief Network não supervisionado e o método de codificação automática (denoising) para prever a qualidade da água usando 3 parâmetros ( $\mathrm{pH}$, Turbidez e OD) como entrada de dados. Para comparar os resultados do modelo desenvolvido foram utilizados os modelos de regressão linear e perceptron multi-camada e concluiu que o modelo 
proposto apresenta melhor resultado de previsão quando o parâmetro apresenta alta variabilidade no conjunto de dados.

[Heddam 2016] comparou a capacidade dos modelos Radial Basis Function Neural Network (RBFNN) e Multilayer Rede Neural Perceptron (MLPNN) para modelagem simultânea e previsão de concentração de $\mathrm{OD}$, utilizando variáveis $(\mathrm{pH}, \mathrm{TE}, \mathrm{SC}$ e SD) da qualidade da água e valores antecedentes de DO. Conseguiu prever com até 72 horas a frente a concentração de OD, mas ressaltou que para um atingir um melhor desempenho é necessária uma base de dados com mais de 1 ano de dados.

[Dragoi et al. 2018] experimentou a modelagem combinada de redes neurais com algoritmo evolutivo para prever a qualidade da água superficial. Foi comparado dois modelos com número de saídas diferentes, concluindo que o modelo com saída única é mais adequada.

Como observado, modelos de redes neurais como MLP, ANFIS e Deep Belief Network foram utilizados para predizer os parâmetros da qualidade da água, avaliando-os usando métricas de erro como erro quadrático médio (RMSE) e erro médio percentual absoluto (MAPE), assim como, comparando o desempenho entre modelos como a regressão linear e perceptron multicamadas. Diante disso, surge a oportunidade de aplicar redes neurais com aprendizado profundo do tipo LSTM para prever no tempo os parâmetros da qualidade da água, avaliando a variação do número de neurônios nas camadas da rede.

\section{Metodologia}

\subsection{Análise do conjunto de dados}

O conjunto de dados utilizado neste artigo refere-se ao monitoramento dos parâmetros da qualidade da água nas UGRHI 10 e 6, as quais chamaremos A e B, respectivamente. Esses dados foram obtidos do Sistema de Informações InfoÁGUAS desenvolvido pela Companhia Ambiental do Estado de São Paulo (CETESB). A periodicidade de monitoramento é mensal abrangendo o período de 01 de janeiro de 1977 à 31 de dezembro de 2019, contabilizando 42 anos.2. A Tabela 1 apresenta a análise estatística dos valores dos parâmetros físicos-químicos da qualidade da água que serão utilizados nos experimentos do trabalho. Ademais, a coluna Contagem representa o número de amostras coletadas mensalmente na UGRHI A.

Tabela 1. Análise estatística da série temporal dos valores dos parâmetros.

\begin{tabular}{l|c|c|c|c|c}
\hline \hline Parâmetro & Contagem & Média & Desvio Padrão & Mínimo & Máximo \\
\hline \hline DBO & 446 & 19.52 & 12.21 & 2.9 & 93.38 \\
Fósforo & 442 & 1.64 & 14.12 & 0.02 & 290.25 \\
OD & 445 & 3.75 & 1.86 & 0.0 & 8.45 \\
pH & 443 & 6.97 & 0.35 & 5.6 & 7.9 \\
Turbidez & 445 & 68.2 & 209.46 & 1.0 & 3416.5 \\
\hline \hline
\end{tabular}

Os gráficos apresentados na Figura 1, demonstra o comportamento da série temporal de cada UGRHI, considerando a média trimestral e anual do valor do parâmetro

${ }^{2}$ Os dados e códigos desse trabalho encontram-se disponíveis online no seguinte endereço https: //github.com/andersonchico/predicaoagua 
pH. Este parâmetro foi selecionado para exemplificar o comportamento da série temporal nas UGRHI A e B devido apresentar o menor desvio-padrão em relação aos demais parâmetros, permitindo observar que os conjuntos de dados das UGRHI abrangem todo período da série temporal, por isso, serão utilizados nos experimentos das predições usando redes neurais LSTM e MLP, comparando os resultados de desempenho quando configuradas com diferentes números de neurônios. Justifica-se o uso da rede neural LSTM, devido possuir a característica de modelar sequencias de longo prazo, como os dados de séries temporais [Yamak et al. 2019].
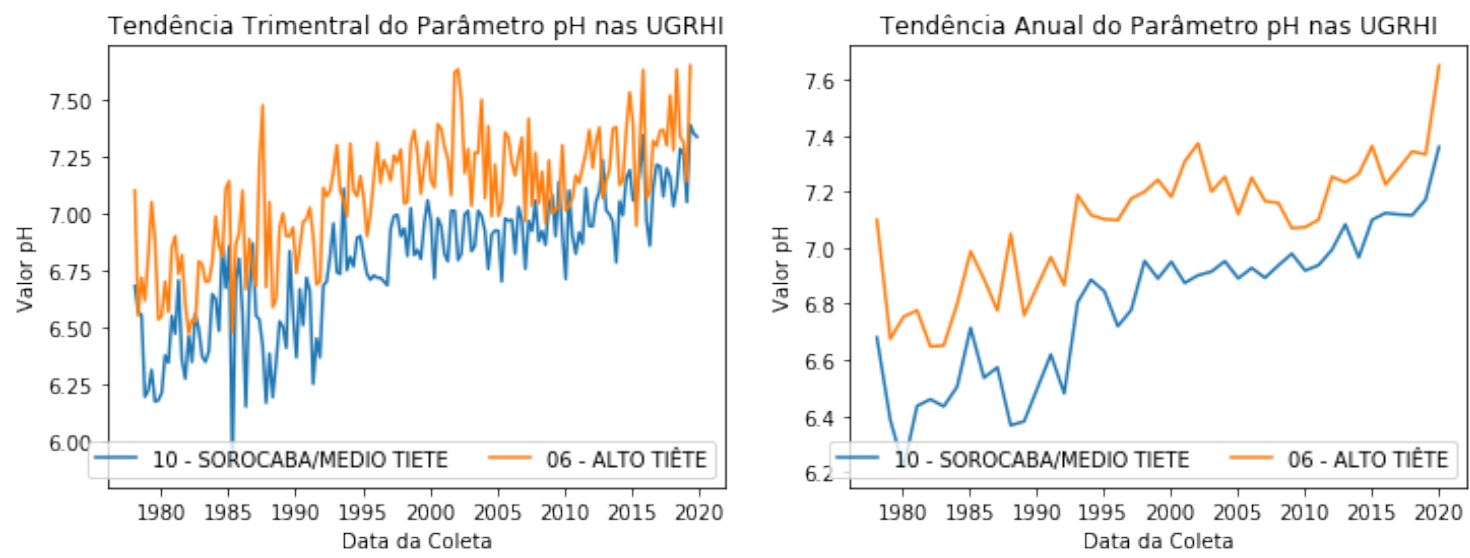

Figura 1. Esq. Média trimestral do Parâmetro pH em cada uma das UGRHI. Dir. Média anual do Parâmetro pH em cada uma das UGRHI.

\subsubsection{Pré-processamento de dados}

Nesta etapa, considerando os dados das UGRHI A e B, foram executados os seguintes procedimentos: a limpeza dos dados, para a retirada de valores ausentes; a transformação da frequência das séries temporais para trimestral, calculando-se a média do valor a cada 3 meses, pois a média mensal apresentou 60 valores ausentes nos dados da UGRHI B e na média bimestral apresentou 3 valores ausentes nos dados da UGRHI A, para cada parâmetro; e a normalização dos valores dos parâmetros utilizando a normalização minmax. Dessa forma, a série temporal trimestral apresenta 166 observações.

\subsubsection{Configurações dos experimentos}

Para predizer as séries temporais, optou-se por treinar as redes neurais utilizando $80 \%$ do conjunto de dados e $20 \%$, da mesma UGRHI, para o teste de validação com 1 trimestre a frente. Em total foram 133 observações para treino, equivalentes ao período de 1977 à 2010. E 33 observações para teste, equivalentes ao período de 2011 a 2019. O intervalo de tempo para predizer os valores dos parâmetros foi definido com 8 observações ( 8 variáveis de entrada), equivalente a 2 anos de amostras.

Para treinamento a rede LSTM foi configurada da seguinte forma: 1 camada LSTM com $x$ neurônios na camada de entrada, 2 camadas LSTM intermediárias com a mesma quantidade de neurônios da camada de entrada, 1 neurônio na camada de saída. 
A rede MLP foi configurada usando só uma camada densa de 10 neurônios e uma camada de saída de um neurônio. Ambas redes neurais foram treinadas com 100 épocas para o ajuste dos pesos sinápticos.

A definição de épocas constante tem o objetivo de manter a igualdade de cenários na avaliação do desempenho das redes neurais, especificamente, quanto aos números de neurônios na camada LSTM. Nos experimentos, a função de perda MSE e RMSE foram utilizados para calcular a variação de erro no treinamento da rede e o otimizador adam com taxa de aprendizado padrão de 0.001 para realizar o ajuste dos pesos baseando-se na variação dos erros.

Para avaliação comparativa entre os modelos, considerou-se verificar o desempenho por número de neurônios e por parâmetros. Na primeira situação, observou-se a média dos erros das redes neurais com diferentes números de neurônios (10, 25 e 50). Na segunda situação, observou-se a média dos erros das redes neurais para cada parâmetro da qualidade da água.

\section{Resultados}

Nesta seção, são analisados os resultados das predições obtidos pelos experimentos com 10, 25 e 50 neurônios nas camadas da rede neural LSTM e MLP.

Observou-se em relação ao desempenho dos parâmetros que pH, OD e Fósforo apresentam melhor desempenho com 50 neurônios nas camadas LSTM. Para o DBO, o melhor desempenho, é quando o modelo está configurado com 25 neurônios. No caso do parâmetro Turbidez houve retorno do tipo $N a N$, mesmo com a redução da taxa de aprendizado do otimizador adam e substituído pelo otimizador RMSprop com valor padrão de 0.001. Este otimizador divide a taxa de aprendizado pela média da deterioração exponencial dos gradientes quadrados [khandelwal 2019]. A Figura 2, apresenta o comparativo das predições dos parâmetros pH, OD, Turbidez e DBO entre os modelos LSTM e MLP, ambos, configurados com 10 neurônios. Pode-se observar que ambos os modelos acompanham a tendência dos valores dos parâmetros tanto em treinamento quanto nos testes. Porém, as predições do modelo LSTM situa-se dentro da média da série temporal, enquanto que as predições do modelo MLP situa-se acima da média. O comportamento do modelo LSTM no experimento, pode ser justificado pela capacidade de armazenar informações importantes do conjunto de dados a longo prazo [Bandara et al. 2020].

Quando considerado o MSE médio como métrica de desempenho, o resultado do parâmetro Fósforo obteve menor MSE médio, 0.00, caracterizando sobreajuste na predição. Os demais parâmetros pH, OD, DBO e Turbidez apresentaram MSE médios de 0.02, 0.03, 0.09 e 0.01, respectivamente. Quanto ao MSE médio relacionado ao número de neurônios nas camadas LSTM, quando configurado com 25 neurônios obteve o menor MSE médio, de 0.034, enquanto que com 10 e 50 neurônios apresentaram MSE médios de 0.036 e 0,035, respectivamente, conforme Figura 3 .

Com modelo LSTM a predição obteve um MAPE médio de 13.49, o parâmetro Fósforo obteve menor RMSE médio, de 0.01, enquanto que os demais parâmetros $\mathrm{pH}$, OD, DBO e Turbidez apresentaram RMSE médios de 0.14, 0.17, 0.26 e 0.08, respectivamente. Quanto ao RMSE médio relacionado ao número de neurônios nas camadas LSTM, quando executado com 25 neurônios obteve o menor RMSE médio, de 0.132, 

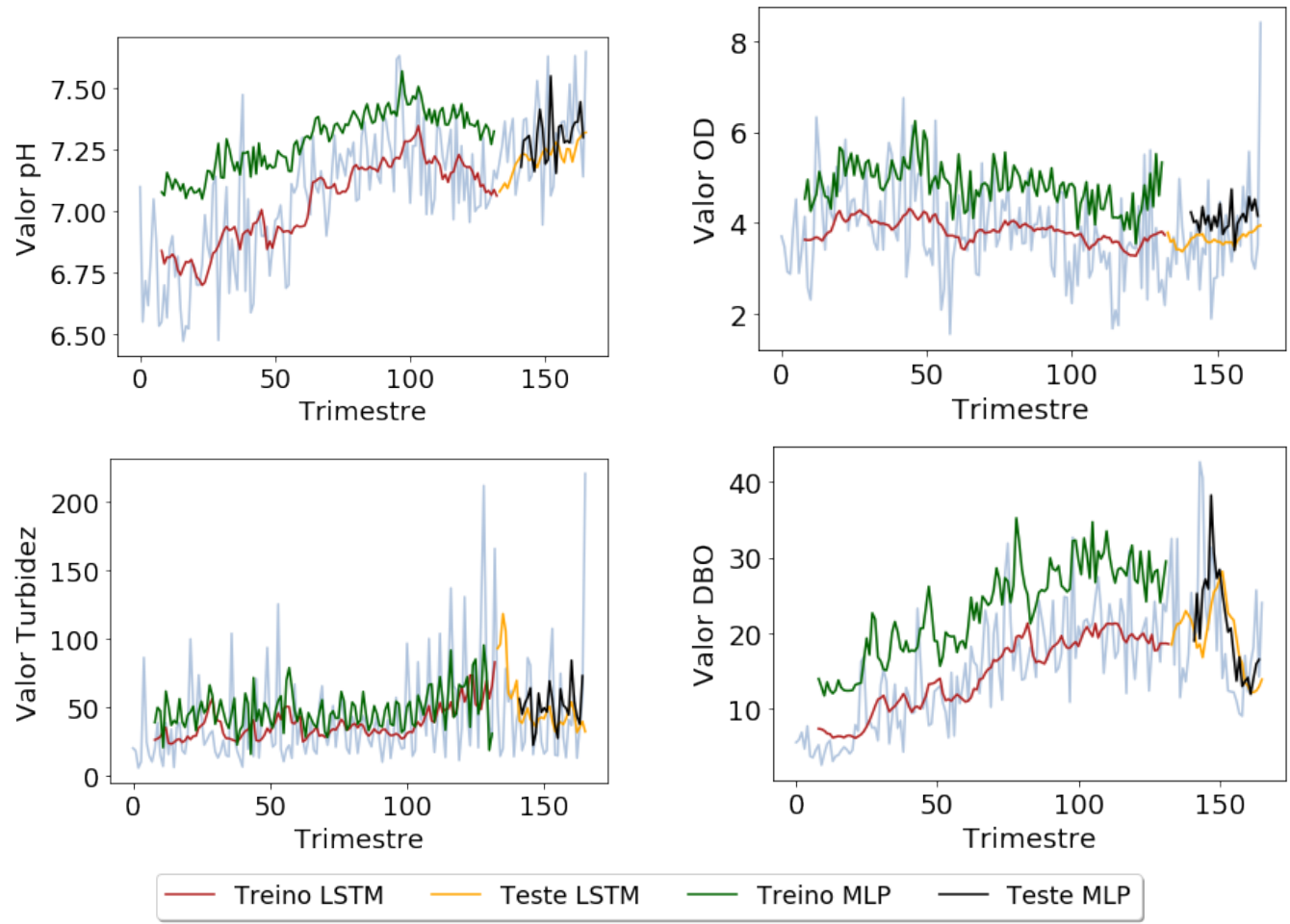

Figura 2. Predição temporal dos valores dos parâmetros pH, OD, Turbidez e DBO na UGRHI A com 10 neurônios nas camadas da rede LSTM e MLP.

enquanto que com 10 e 50 neurônios apresentaram RMSE médios de 0.137 e 0,134, respectivamente. Com o modelo MLP a predição obteve um MAPE médio de 13.03, o parâmetro Fósforo obteve menor RMSE médio, de 0.00, caracterizando sobreajuste na predição, enquanto que os demais parâmetros pH,OD,DBO e Turbidez apresentaram RMSE médios de 0.12, 0.11, 0.15 e 0.04, respectivamente. Quanto o RMSE médio relacionado ao número de neurônios nas camadas do modelo MLP, quando executado com 50 neurônios obteve o menor RMSE médio, de 0.076, enquanto que com 10 e 25 neurônios apresentaram RMSE médios de 0.095 e 0,084, respectivamente.

Na Figura 4, o gráficos apresentam a comparação de desempenho entre os modelos LSTM e MLP, em relação ao número de neurônios e quanto aos parâmetros $\mathrm{pH}$, OD, DBO, Fósforo e Turbidez. Pode-se observar que o modelo MLP obteve o menor RMSE médio, quando o desempenho é relacionado ao número de neurônios nas camadas dos modelos, assim como, para os parâmetros pH, OD, DBO e Turbidez. Quanto ao parâmetro Fósforo, devido sobreajuste do modelo MLP, que é alta adaptação ao do conjunto de treinamento perdendo a capacidade de generalização [Santos et al. 2005], portanto, consideramos que o modelo LSTM apresentou melhor desempenho de predição do parâmetro.

\section{Conclusões e Trabalhos Futuros}

Este artigo apresentou os seguintes comparativos de desempenho: 1) entre modelos de rede neural LSTM quando configuradas com 10, 25 e 50 neurônios nas camadas da rede para predizer os parâmetros $\mathrm{pH}, \mathrm{OD}, \mathrm{DBO}$, Fósforo e Turbidez da qualidade da água, utilizando como métrica de desempenho o erro quadrático médio (MSE); 2) entre o modelo 

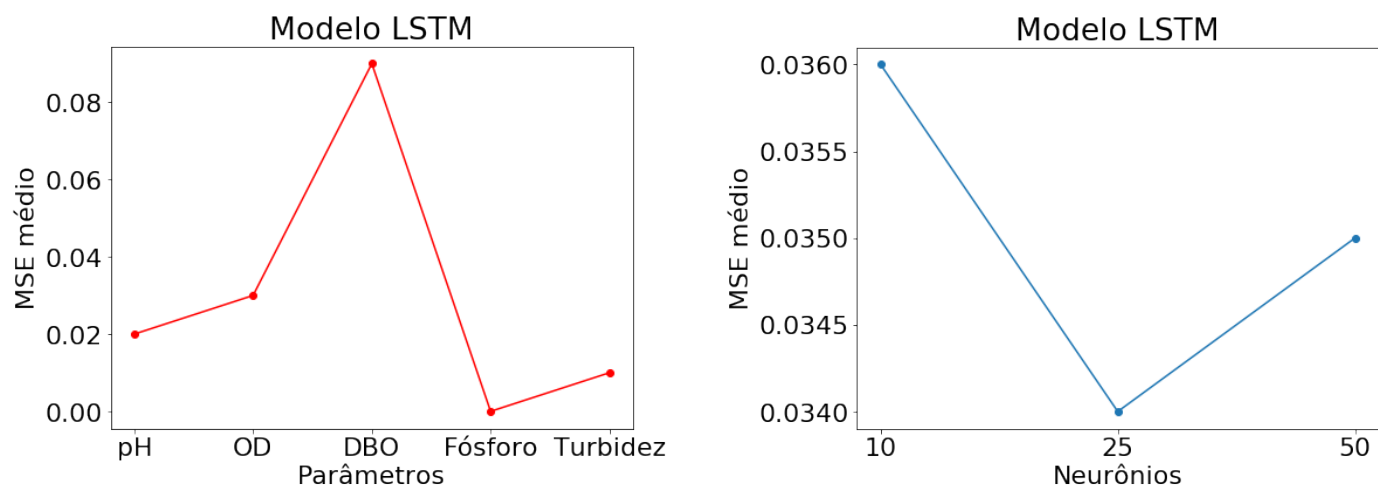

Figura 3. Esq. MSE médio do modelo LSTM em relação aos parâmetros. Dir. MSE médio do modelo LSTM em relação ao no de neurônios.

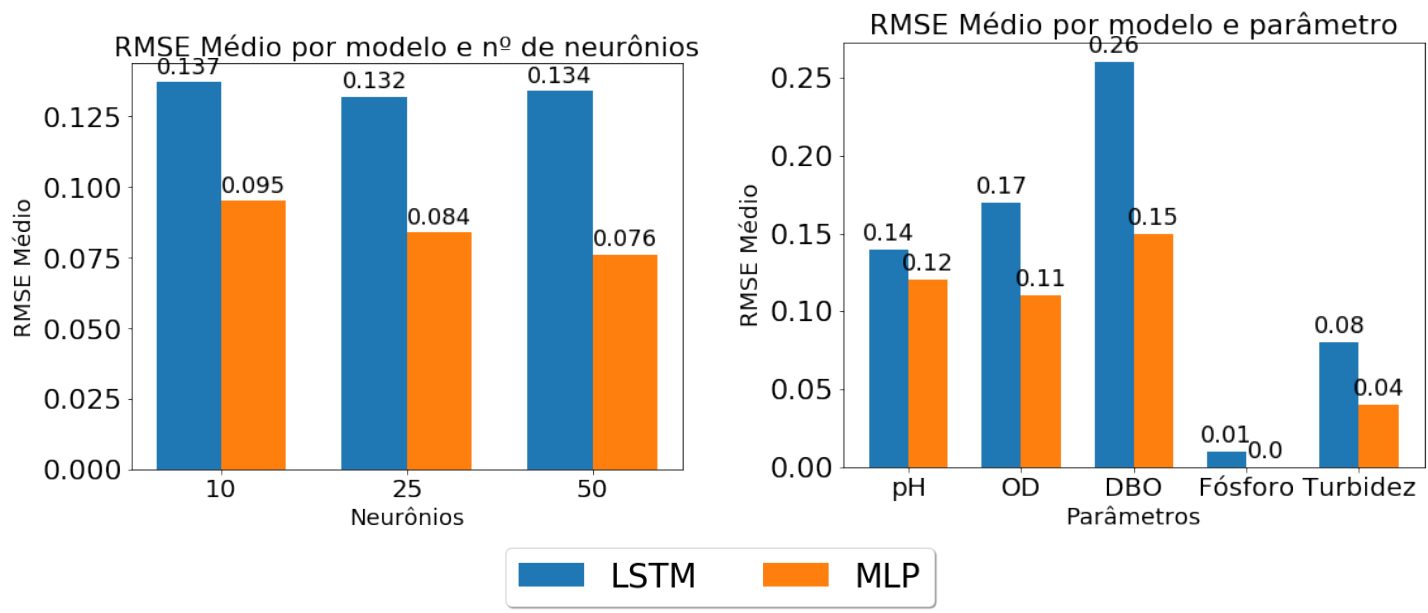

Figura 4. Esq. Comparativo de RMSE médio dos modelos LSTM e MLP em relação ao no de neurônios. Dir. Comparativo de RMSE médio dos modelos LSTM e MLP em relação aos parâmetros.

LSTM e MLP, ambos com a mesma configuração, entretanto utilizando como métrica de desempenho a raiz do erro quadrático médio (RMSE).

Dessa forma, nos experimentos com a rede LSTM, os resultados demonstram melhor desempenho quando configurada com 25 neurônios, na qual o MSE médio é de 0.034,o parâmetro fósforo apresentou sobreajuste na predição com MSE médio de 0.00. Quanto a comparação entre os modelos LSTM e MLP, o último modelo apresentou melhor desempenho em todos os cenários com 10, 25 e 50 neurônios, assim como, quando comparado o desempenho dos modelos por parâmetro.

Como trabalhos futuros cria-se a expectativa de utilizar os mesmos conjuntos de dados para predizer espacialmente os parâmetros pH, OD, DBO, Fósforo e Turbidez da qualidade da água. Outras técnicas de aprendizado de máquina com menor complexidade como regressão linear, máquinas de vetor de suporte e random forest poderiam ser usadas nessas comparações. 


\section{Referências}

ANA (2018). Conjuntura dos recursos hídricos no brasil 2018: relatório pleno. Technical report, Agência Nacional de Águas.

Bandara, K., Bergmeir, C., and Smyl, S. (2020). Forecasting across time series databases using recurrent neural networks on groups of similar series: A clustering approach. Expert Systems with Applications, 140:112896.

Da Silva, I. N., Spatti, D. H., and Flauzino, R. A. (2010). Redes NEurais Artificiais para Engenharia e Ciências Aplicadas: Curso Prático. Artliber.

de Fravet, A. M. M. F. and Cruz, R. L. (2007). Qualidade da água utilizada para irrigação de hortaliças na região de botucatu-sp. Irriga, pages 144-155.

Derisio, J. C. (2016). Introdução ao controle de poluição ambiental. Oficina de Textos.

Dragoi, E., Kovács, Z., Juzsakova, T., Curteanu, S., and Cretescu, I. (2018). Environmental assesment of surface waters based on monitoring data and neuro-evolutive modelling. Process Safety and Environmental Protection, 120:136-145.

Gastaldini, M., Mendonça, A., Paiva, J., and Paiva, E. (2001). Conceitos para a avaliação da qualidade da água. PAIVA, JBD; PAIVA, EMCD Hidrologia aplicada à gestão de pequenas bacias hidrográficas. Porto Alegre: $A B R H$, pages 428-51.

Gastaldini, M. and Teixeira, E. (2001). Avaliação da qualidade da água. PAIVA, JBD; PAIVA, EMCD Hidrologia aplicada à gestão de pequenas bacias hidrográficas. Porto Alegre: ABRH, pages 453-90.

Goldschmidt, R. and Passos, E. (2005). Data mining: um guia Prático. Elsevier.

Haykin, S. (2007). Redes neurais: princípios e prática. Bookman Editora.

Heddam, S. (2016). Simultaneous modelling and forecasting of hourly dissolved oxygen concentration (do) using radial basis function neural network (rbfnn) based approach: a case study from the klamath river, oregon, usa. Modeling Earth Systems and Environment, 2(3):135.

khandelwal, R. (2019). Overview of different optimizers for neural networks.

Monard, M. C. and Baranauskas, J. A. (2003). Conceitos sobre aprendizado de máquina. Sistemas inteligentes-Fundamentos e aplicações, 1(1):1.

Olah, C. (2015). Understanding lstm networks, 2015. URL http://colah. github. io/posts/2015-08Understanding-LSTMs.

Sahoo, M. M., Patra, K., and Khatua, K. (2015). Inference of water quality index using anfia and pca. Aquatic Procedia, 4:1099-1106.

Santos, A. M. d., Seixas, J. M. d., Pereira, B. d. B., and Medronho, R. d. A. (2005). Usando redes neurais artificiais e regressão logística na predição da hepatite a. Revista Brasileira de Epidemiologia, 8(2):117126.

Sarkar, A. and Pandey, P. (2015). River water quality modelling using artificial neural network technique. Aquatic Procedia, 4:1070-1077.

Solanki, A., Agrawal, H., and Khare, K. (2015). Predictive analysis of water quality parameters using deep learning. International Journal of Computer Applications, 125(9):0975-8887.

Yamak, P. T., Yujian, L., and Gadosey, P. K. (2019). A comparison between arima, 1stm, and gru for time series forecasting. In Proceedings of the 2019 2nd International Conference on Algorithms, Computing and Artificial Intelligence, ACAI 2019, page 49-55, New York, NY, USA. Association for Computing Machinery. 